\title{
Composability and On-Line Deniability of Authentication
}

\author{
Yevgeniy Dodis ${ }^{1, \star}$, Jonathan $\mathrm{Katz}^{2, \star \star}$, Adam $\mathrm{Smith}^{3, \star \star \star}$, \\ and Shabsi Walfish ${ }^{4, \dagger}$ \\ ${ }^{1}$ Dept. of Computer Science, New York University \\ 2 Dept. of Computer Science, University of Maryland \\ ${ }^{3}$ Dept. of Computer Science and Engineering, Pennsylvania State University \\ ${ }^{4}$ Google, Inc.
}

\begin{abstract}
Protocols for deniable authentication achieve seemingly paradoxical guarantees: upon completion of the protocol the receiver is convinced that the sender authenticated the message, but neither party can convince anyone else that the other party took part in the protocol. We introduce and study on-line deniability, where deniability should hold even when one of the parties colludes with a third party during execution of the protocol. This turns out to generalize several realistic scenarios that are outside the scope of previous models.

We show that a protocol achieves our definition of on-line deniability if and only if it realizes the message authentication functionality in the generalized universal composability framework; any protocol satisfying our definition thus automatically inherits strong composability guarantees. Unfortunately, we show that our definition is impossible to realize in the PKI model if adaptive corruptions are allowed (even if secure erasure is assumed). On the other hand, we show feasibility with respect to static corruptions (giving the first separation in terms of feasibility between the static and adaptive setting), and show how to realize a relaxation termed deniability with incriminating abort under adaptive corruptions.
\end{abstract}

\section{Introduction}

Message authentication allows a sender $S$ to authenticate a message $m$ to a receiver $R$. If $S$ has a public key, message authentication is usually handled using digital signatures. A well-known drawback of digital signatures, however, is that they leave a trace of the communication and, in particular, allow $R$ (or, in fact, any eavesdropper) to prove to a third party that $S$ authenticated the message in question. In some scenarios such non-repudiation is essential, but in many other cases deniability is desired.

The original version of this chapter was revised: The copyright line was incorrect. This has been corrected. The Erratum to this chapter is available at DOI: 10.1007/978-3-642-00457-5_36

* Supported by NSF grants CNS-0831299, CNS-0716690, CCF-0515121, and CCF0133806. A portion of this work was done while visiting CRCS at Harvard University.

** Supported by NSF CNS-0447075 and NSF CNS-0627306.

*** Supported by NSF TF-0747294 and NSF CAREER award 0729171.

${ }^{\dagger}$ A portion of this work was done while at New York University.

O. Reingold (Ed.): TCC 2009, LNCS 5444, pp. 146-162, 2009.

(C) Springer-Verlag Berlin Heidelberg 2009 
Deniable authentication, introduced in [16, 18] and studied extensively since then, achieves the seemingly paradoxical guarantees that (1) the receiver is convinced that the message originated from the sender, yet (2) the receiver, even if malicious, cannot prove to anyone else that the sender authenticated the given message. Furthermore, (3) the receiver cannot be incriminated as having been involved, even by a malicious sender (this is meaningful when the receiver has a public key, as will be the case in our work; see further below).

Deniability is a fundamental concept in cryptography. Non-repudiation is sometimes crucial for the free exchange of ideas: without the assurance of remaining "off the record", individuals may be discouraged from discussing subversive (or embarrassing) topics. Deniability is also intimately tied to the simulation paradigm that is central to our understanding of cryptographic protocols.

Indeed, deniability is typically formalized via the simulation paradigm introduced in the context of zero-knowledge (ZK) proofs 20]. Zero-knowledge proofs, however, do not automatically provide deniability. Pass 27 points out that non-interactive ZK proofs are not deniable, nor are many existing ZK proofs in the random oracle model. Furthermore, ZK proofs for which simulation requires rewinding may not suffice to achieve on-line deniability which protects each party even when the other party colludes with an on-line entity that cannot be "rewound" (see below for an example)1 Looking ahead, we note that on-line deniability is only potentially feasible if receivers hold public keys, and we assume this to be the case in our work. Once receivers have public keys, however, protocols can realize the stronger semantics by which a sender can authenticate a message for a specific receiver $R$ but not for anyone else.

One might question whether on-line deniability is too strong. To see why it might be essential, consider a setting where Bob talks to Alice while relaying all messages to/from an external party (such as a law-enforcement agent). Ideally, a deniable authentication protocol would not permit the agent to distinguish the case when Bob is having a real conversation with Alice from the case when Bob is fabricating the entire interaction. Previous, off-line models of deniability provide no guarantees in this setting. Alternatively, imagine a publicly readable/writeable bulletin board (e.g., a wiki) where all entries are time-stamped and assigned unpredictable identifiers. A corrupt receiver running a protocol with an honest sender can post all the messages it receives to the bulletin board, and then generate its responses based on the identifiers assigned to the resulting posts. Again, off-line deniability would not suffice since the bulletin board cannot be rewound; in this case, the contents of the bulletin board would prove that the interaction occurred. More generally, one can imagine a "chosen-protocol attack" by which someone designs and deploys a public service specifically targeted to destroying the deniability of a particular authentication protocol.

With the above motivation in mind, we introduce a strong notion of deniability that, in particular, implies on-line deniability. We then show that a protocol

${ }^{1}$ In contrast, previous notions of deniable authentication only guarantee off-line deniability which protects against a malicious party who records the transcript and shows it to a third party after the fact. 
satisfies our definition if and only if it securely realizes the message authentication functionality $\mathcal{F}_{\text {auth }}$ in the recently introduced generalized $U C$ (GUC) framework 7]. (This is an extension of Canetti's UC framework [5] that models globally-available, "external" functionalities like a common reference string, PKI, etc.) Protocols proven secure with respect to our definition thus inherit all the strong composability properties of the $(\mathrm{G}) \mathrm{UC}$ framework. We stress that protocols realizing $\mathcal{F}_{\text {auth }}$ in the UC framework do not necessarily provide deniability; in particular, digital signatures — which are clearly not deniable realize $\mathcal{F}_{\text {auth }}$ in the UC framework 6]. Similarly, protocols realizing $\mathcal{F}_{\text {auth }}$ in the UC framework may be problematic when composed with other protocols that are allowed to depend on parties' public keys (see Section [2.3). In both cases, the reason is that the $\mathrm{UC}$ framework treats public keys as local to a particular session. When this condition is enforced, the expected security properties hold; when public keys are truly public, the expected security properties may not hold.

\subsection{Our Results}

We propose a definition of deniable authentication which, in comparison to prior work, guarantees stronger security properties such as on-line deniability and security under concurrent executions with arbitrary other protocols. Unfortunately, we show that our notion of deniable authentication is impossible to achieve in the PKI model if adaptive corruptions are allowed. This holds even if secure erasure is assumed; if we are unwilling to allow erasure then we can rule out even the weaker notion of forward security (where, informally, honest parties' secret keys and state might be compromised after completion of the protocol). In the full version, we show reductions from deniable authentication to deniable key exchange and vice versa; thus, our impossibility results imply that deniable key exchange is impossible (with regard to adaptive corruptions) as well.

Our impossibility result is very different from prior impossibility results in the UC setting [5, 9, 11,7, Previous impossibility results assume secure channels as a primitive, and show that additional setup assumptions (such as a PKI) are necessary to realize other, more advanced functionalities. Here, we show that the basic functionality of authenticated channels cannot be realized even given the setup assumption of a PKI.

Faced with this strong negative result, we ask whether relaxed definitions of deniable authentication can be achieved. In this direction, we show several positive results based on standard assumptions and without random oracles. First, we observe that our definition can be satisfied with respect to static adversaries. This appears to give the first separation between the static and adaptive settings with regard to feasibility 2 Second, we observe that our definition can be achieved, with respect to adaptive corruptions, in the symmetric-key setting where all pairs of parties share a key.

\footnotetext{
${ }^{2}$ Nielsen [26] shows a separation between the static and adaptive settings with regard
} to round complexity, but not feasibility. 
The symmetric-key setting is less appealing than the public-key setting. To partially bridge the two we suggest that symmetric keys for deniable authentication can be established using a weak form of deniable key exchange termed key exchange with incriminating abort (KEIA). Intuitively, KEIA guarantees deniability as long as the protocol terminates successfully; once a shared key is established, deniability is guaranteed even if corruptions occur at any later time. If a malicious party aborts the protocol, however, this party may obtain some incriminating evidence against the other party; all this proves, however, is that the two parties attempted to establish a key. In light of our impossibility result, realizing KEIA (and hence a weak form of deniable authentication) seems to be a reasonable compromise.

As our third and most technically interesting feasibility result, we show how to realize KEIA in the PKI model (without erasure) with respect to semi-adaptive adversaries who corrupt parties either before or after (but not during) an execution of the protocol.

Due to space constraints, this extended abstract discusses the proposed modeling of online deniability and states our main results. Proofs, additional results, and further discussions are deferred to the full version.

\subsection{Previous Work in Relation to Our Own}

Deniable authentication was formally introduced by Dwork, Naor, and Sahai 18 (though it was also mentioned in [16]) and it, along with several extensions and generalizations, has received significant attention since then 4, 31, 3, 18, 19, 17, 24, 27, 28, 14, 7, 25. This prior work all assumes that only the sender has a public key; thus, this work implicitly assumes that "guaranteed delivery" of messages to a specific, intended recipient is possible, and/or that the sender is willing to authenticate a given message for anyone. In such cases on-line deniability does not make sense. Since we are specifically interested in on-line deniability, we consider the setting where the receiver also has a public key. (This setting was also considered in concurrent work done independently of our own [23, 32.) Once the receiver holds a public key, the sender can meaningfully authenticate a message for a particular receiver without being willing to authenticate the message for all other parties.

As previously mentioned, our definition implies very strong notions of deniability. In particular, our protocols remain secure under concurrent composition, something that was an explicit goal of prior work 18, 19, 24, 28, To the best of our knowledge all prior constructions achieving concurrent security use timing assumptions [18] which, though reasonable, seem preferable to avoid 3 Our protocols also remain secure when run concurrently with arbitrary other protocols, something not addressed by previous work.

Designated-verifier proofs [22] and two-party ring signatures [30,2] also provide authentication without non-repudiation. These primitives do not provide

${ }^{3}$ It is not clear whether plugging a generic concurrent ZK proof [29] into any existing deniable authentication protocol would yield a concurrently secure protocol. In any case, this approach would yield protocols with very high round complexity [10]. 
deniable authentication, however, since they incriminate the sender $S$ and receiver $R$ jointly; that is, they $d o$ leave evidence that either $S$ or $R$ was involved in authenticating some message. Deniable authentication, in contrast, does not implicate either party. Similarly, although there has been extensive work constructing and analyzing various deniable key-exchange protocols such as as SIGMA, SKEME, and HMQV (see 13, 14), none of these protocols meets our definition of deniability. For example, SIGMA leaves a trace that the sender and receiver communicated, even if it does not reveal exactly what message was authenticated. (HMQV might satisfy our definition with respect to static adversaries, though we have not verified the details. The HMQV protocol is not, however, forward-secure unless erasure by honest parties is allowed).

\section{Defining Deniable Authentication}

In this section we define our notion of deniable authentication. We begin by giving a self-contained definition whose primary aim is to model on-line deniability. Our definition is based on an interactive distinguisher, much like the "environment" in the UC framework. Indeed, we observe that a protocol satisfies our definition if and only if it securely realizes the message authentication functionality $\mathcal{F}_{\text {auth }}$ in the GUC framework. This means that any protocol satisfying our definition automatically inherits the strong composability guarantees of the UC framework, and also provides some justification of the claim of Canetti et al. [7] that the GUC-framework models deniability.

\subsection{The Basic Definition}

We start by introducing the relevant parties. We have a sender $S$ who is presumably sending a message $m$ to a receiver $R$, a judge $\mathcal{J}$ who will eventually rule whether or not the transmission was attempted, an informant $\mathcal{I}$ who witnesses the message transmission and is trying to convince the judge, and a misinformant $\mathcal{M}$ who did not witness any message transmission but still wants to convince the judge that one occurred. Jumping ahead, a protocol is secure if the judge is unable to distinguish whether it is talking to a true informant $\mathcal{I}$ (interacting with $S$ and $R$ while they are running the protocol), or a misinformant $\mathcal{M}$.

We assume the sender and receiver are part of a network environment that includes some trusted parties (e.g., trusted setup like the PKI), some means of communication between the sender and receiver (e.g., a direct unauthenticated channel), and a direct, private channel between the judge and the informant (or misinformant, depending on the setting). Intuitively, this on-line channel, coupled with the fact that $\mathcal{J}$ cannot be "rewound", is what guarantees on-line deniability. Additionally, we assume that the judge does not have direct access to the players (in particular, the judge does not know whether $S$ really intends to send a message, or whether $R$ really received one); instead, the judge must obtain information about the parties through the (mis)informant. However, the judge $\mathcal{J}$ does have direct access to any global setup (for example, in the case of a PKI it can reliably obtain the public keys of $S$ and $R$ ), and so the misinformant 
cannot necessarily lie arbitrarily without being caught. Both the informant $\mathcal{I}$ and the misinformant $\mathcal{M}$ can adaptively corrupt either the sender $S$ or the receiver $R$ at any time, and thereby learn the entire state of the corrupted party (if this party has a public key, this state includes the corresponding secret key). Additionally, once either $S$ or $R$ is corrupt the judge learns about the corruption, and the (mis)informant can totally control the actions of this party going forward. We assume the (mis)informant cannot corrupt the global setup; for example, in the case of a PKI, the (mis)informant does not know the secret keys of any uncorrupted party (but does know all the public keys). Finally, the (mis)informant has partial control over the network: it can totally control all unauthenticated links, and can block messages from authenticated links.

Roughly, a protocol $\pi$ achieves on-line deniable authentication if for any efficient informant $\mathcal{I}$, there exists an efficient misinformant $\mathcal{M}$ such that that no efficient judge $\mathcal{J}$ can distinguish the following two experiments with nonnegligible probability.

1. Informant experiment. $S$ and $R$ run $\pi$ in the presence of the informant $\mathcal{I}$ (who in turn interacts with the judge $\mathcal{J}$ in an on-line manner). $R$ informs $\mathcal{J}$ upon accepting any message $m^{\prime}$ as having been authenticated by $S$ (the message $m^{\prime}$ need not be the same as the input to $S$ if, say, $S$ is corrupt and ignores its input).

2. Misinformant experiment. $S$ and $R$ do nothing, and $\mathcal{J}$ only interacts with the misinformant $\mathcal{M}$. (Here, $\mathcal{M}$ is allowed to falsely inform $\mathcal{J}$ that $R$ accepted some arbitrary message $m^{\prime}$ as having been authenticated by $S$.)

(See the full version for a precise definition.) As in the UC model, we can take the informant $\mathcal{I}$ to be a "dummy" attacker who follows the instructions of the judge and truthfully reports everything it sees.

\subsection{Deniable Authentication in the GUC Framework}

The ideal message authentication functionality $\mathcal{F}_{\text {auth }}$ (essentially from [5]) is given in Figure 1. (In all our ideal functionalities, delivery of messages to parties is scheduled by the adversary.) $\mathcal{F}_{\text {auth }}$ is "deniable" because, although the adversary learns that a message transmission took place, the adversary is not provided with any "evidence" of this fact that would convince a third party. Since $\mathcal{F}_{\text {auth }}$ is deniable, we expect that a protocol $\pi$ realizing $\mathcal{F}_{\text {auth }}$ (with respect to a sufficiently strong notion of "realizing") would be deniable as well. Such a claim is not, however, immediate; in particular, recall that protocols realizing $\mathcal{F}_{\text {auth }}$ in the UC framework are not necessarily deniable.

Thus, we turn instead to a recently-proposed extension to the UC framework called generalized universal composability (GUC) [7] which enables direct modeling of global setup. Canetti et al. claim [7, informally, that modeling global setup in this way provides a means of capturing additional security concerns, including deniability, within a UC-style security framework. We validate their claim (at least in our context) via the following result:

Proposition 1. A protocol $\pi$ achieves on-line deniable authentication if and only if it realizes $\mathcal{F}_{\text {auth }}$ in the GUC framework. 


\section{Functionality $\mathcal{F}_{\text {auth }}$}

1. Upon receiving input (send, sid, $m$ ) from $S$, do: If sid $=\left(S, R, \operatorname{sid}^{\prime}\right)$ for some $R$, then give the message (sent, sid, $m$ ) to the adversary who then schedules delivery to $R$. Else ignore the input.

2. Upon receiving (corruptsend, sid, $m^{\prime}$ ) from the adversary, if $S$ is corrupt and no message (sent, sid, $m$ ) was yet output, then give the message (sent, sid, $m^{\prime}$ ) to the adversary who then schedules delivery to $R$.

Fig. 1. The message authentication functionality of [5]

In the full version of this paper we define notions of on-line deniability for identification and key exchange, and show that protocols achieve these definitions if and only if they realize appropriate functionalities in the GUC framework.

\subsection{PKI Setup and Comparison with Prior Models}

We model a PKI as a shared functionality $\mathcal{F}_{k r k}$ that enforces the following: Honest parties register with a central authority who generates the public and secret keys for them. Corrupt parties register an arbitrary, but consistent, pair of public/secret keys with the authority. Note that the central registration authority knows the secret keys of all parties (including the corrupt parties), and therefore the model is referred to as "Key Registration with Knowledge" 11. Clearly this model is more involved than a "bare" PKI. The fact that we work in this model only strengthens our impossibility results. Moreover, the bare PKI model does not suffice for our feasibility results (cf. Proposition [2).

An additional requirement we impose is that honest parties protect their secret keys by using them only in some specified authentication protocol $\Phi$. (Corrupt parties are allowed to use their keys in an arbitrary manner.) There are several ways to model this requirement. For concreteness, we parameterize the keyregistration functionality with a description of $\Phi$, and allow honest parties to run $\Phi$ via calls to the key-registration functionality. (See Figure 21) In a real execution of the protocol, of course, honest parties will actually hold their secret key and it is up to them to restrict its use.

Comparison to prior models of a PKI. Our PKI functionality is defined similarly to that of 1]. However, unlike in [1, we restrict honest parties to only use their secret keys with the protocol $\Phi$, and a single instance of $\mathcal{F}_{k r k}$ persists across multiple sessions. In the terminology of $\left[7, \mathcal{F}_{k r k}\right.$ is a shared functionality as opposed to a local one. The shared nature of the functionality implies, in particular, that the environment has direct access to the public keys of all the parties (as well as the secret keys of corrupted parties). Local setup, in contrast, is not adequate for capturing deniability. As argued in 7], local setup is also not satisfactory with regard to composition. Indeed, local modeling of the PKI seemingly leaves two options: either a fresh instance of the PKI is required for every execution of the protocol (which is impractical), or one must use the joint UC (JUC) theorem [12. Unfortunately, the latter option only guarantees security under composition with a restricted class of protocols. Specifically, security is 


\section{Shared Functionality $\mathcal{F}_{k r k}^{\Phi}$}

Parameterized by a security parameter $\lambda$, a protocol (or, more generally, a list of protocols) $\Phi$, and a (deterministic) key generation function Gen, shared functionality $\mathcal{F}_{k r k}$ proceeds as follows when running with parties $P_{1}, \ldots, P_{n}$ :

Registration: When receiving a message (register) from an honest party $P_{i}$ that has not previously registered, sample $r \leftarrow\{0,1\}^{\lambda}$ then compute $\left(P K_{i}, S K_{i}\right) \leftarrow \operatorname{Gen}^{\lambda}(r)$ and record the tuple $\left(P_{i}, P K_{i}, S K_{i}\right)$.

Corrupt Registration: When receiving a message (register, $r$ ) from a corrupt party $P_{i}$ that has not previously registered, compute $\left(P K_{i}, S K_{i}\right) \leftarrow \operatorname{Gen}^{\lambda}(r)$ and record the tuple $\left(P_{i}, P K_{i}, S K_{i}\right)$.

Public Key Retrieval: When receiving a message (retrieve, $P_{i}$ ) from any party $P_{j}$ (where $i=j$ is allowed), if there is a previously recorded tuple of the form $\left(P_{i}, P K_{i}, S K_{i}\right)$, then return $\left(P_{i}, P K_{i}\right)$ to $P_{j}$. Otherwise return $\left(P_{i}, \perp\right)$ to $P_{j}$.

Secret Key Retrieval: When receiving a message (retrievesecret, $P_{i}$ ) from a party $P_{i}$ that is either corrupt or honestly running the protocol code for $\Phi$, if there is a previously recorded tuple of the form $\left(P_{i}, P K_{i}, S K_{i}\right)$ then return $\left(P_{i}, P K_{i}, S K_{i}\right)$ to $P_{i}$. In all other cases, return $\left(P_{i}, \perp\right)$.

Fig. 2. The $\Phi$-Key Registration with Knowledge shared functionality

not guaranteed under composition with protocols that may depend on honest parties' public keys. (We provide an example in the full version.)

\subsection{Flavors of Protocols/Attackers}

An adaptive attacker can corrupt parties before, during, and after execution of a protocol. A static attacker can only corrupt parties before the beginning of the protocol. A semi-adaptive attacker can corrupt parties before and after (but not during) a protocol execution. Finally, a forward-secure attacker can only corrupt the parties after the protocol. We will distinguish between the setting where (honest) parties are assumed to be able to securely erase information, and where they cannot. Erasures do not affect the model for static attackers, but are meaningful for semi-adaptive, forward-secure, and fully adaptive attackers.

\section{Impossibility Result}

In this section we prove our main result: adaptively secure deniable authentication is impossible in the PKI model, even if erasures are allowed, and even if each secret key is used only once. If secure erasure is not assumed, we can even rule out forward security for deniable authentication.

Before stating the precise impossibility results, it is instructive to consider some naïve strategies for ruling out adaptively secure on-line deniable authentication. At first, it appears that since the behavior of the sender $S$ can be simulated in a straight-line manner without its secret key $S K_{S}$, there is an attacker who can impersonate the sender to the recipient $R$ (by running the simulator). 
One of the reasons this does not work is that $R$ might use its own secret key $S K_{R}$ for verification. In particular, a simulated transcript might be easily distinguishable from a real transcript to $R$ (since $R$ can employ knowledge of $S K_{R}$ to distinguish the transcript) but be indistinguishable from a real transcript to the adversary. One "fix" to this problem is for the adversary to (adaptively) corrupt $R$ and then check the simulated transcript from $R$ 's viewpoint. Unfortunately, if $R$ is corrupted too early (say, at the very beginning), it could be the case that knowledge of $R$ 's secret key is subsequently employed by the simulator in order to simulate the proper transcript (without talking to $S$ or obtaining $S K_{S}$ ). Notice that such a simulation does not contradict soundness since, in the real world, $R$ would know that he is not simulating the conversation with $S$. On the other hand, if $R$ is corrupted too late (say, at the very end), the initial flows from the "then-honest" party $R$ were also chosen by the simulator, so there is no guarantee that they correspond to the behavior of a real $R$ interacting with the sender's simulator.

In fact, a proof of the following theorem is more complicated and requires a sequence of "hybrid arguments" where corruption of one of the parties is delayed by one round each time. See the following section.

Theorem 1. There does not exist a protocol $\Pi$ realizing the deniable authentication functionality $\mathcal{F}_{\text {auth }}$ in the $\mathcal{F}_{k r k}^{\Pi}$-hybrid model with respect to adaptive corruptions. Moreover, impossibility holds even under the following additional assumptions/constraints:

- Secure data erasures are allowed.

- Each honest party $P$ uses its secret key sk $k_{P}$ for only a single execution of the protocol.

- The attacker $\mathcal{A}$ either impersonates a sender to a single honest receiver, or impersonates a receiver to a single honest sender. (In particular, $\mathcal{A}$ does not run a concurrent attack or a "man-in-the-middle attack" against an honest sender and receiver.)

We also show how to extend the above impossibility result to rule out forward security if erasures are not allowed.

Theorem 2. If data erasures are not allowed, it is impossible to realize $\mathcal{F}_{\text {auth }}$ in the $\mathcal{F}_{k r k}$-hybrid model with respect to forward security. Moreover, impossibility holds even under the constraints of Theorem 1 .

The results above can also be extended to rule out the possibility of realizing deniable key exchange or identification.

Finally, we note that the "bare PKI" model (in which parties are allowed to post public keys without necessarily knowing a corresponding secret key) is not sufficient to realize deniable authentication at all, even with respect to security. This seems to imply that key registration with knowledge is an unavoidable requirement for deniability.

Proposition 2. It is impossible to realize the identification, authentication, or key exchange functionalities in the bare public key model, even with respect to static corruption. 


\subsection{Proof Sketch for Impossibility (Theorem I)}

At a high level, the proof is an inductive argument showing that each round of the protocol either incriminates one of the parties, or can be simulated entirely (from either side) without knowledge of any secret keys. Of course, if either party can simulate the entire protocol without knowledge of any secret keys, it cannot be sound (i.e., an attacker without $S$ 's key can authenticate an arbitrary message to $R$ ). Thus, we show that either the protocol is not deniable, or it is not sound, contradicting our security requirements. The difficult part of the proof is the inductive step, which requires a delicate series of hybrid arguments. In particular, one must be careful about the order of corruptions in the various hybrids.

More formally, let $\Pi$ be any protocol for deniable identification using $r=r(n)$ rounds, and assume toward a contradiction that $\Pi$ is adaptively secure. Without loss of generality, we assume that the receiver goes first, and that the final message of the protocol is sent by the sender. In particular, we let $\alpha_{1}, \alpha_{2}, \ldots, \alpha_{r}$ denote the messages sent by the receiver $R$ and $\beta_{1}, \ldots, \beta_{r}$ denote the response messages sent by the sender $S$. For convenience, we let $\alpha_{r+1}$ denote the binary decision bit of the receiver indicating whether or not $R$ accepted. Throughout the protocol, we denote the current state of the sender and the receiver by $\omega_{S}$ and $\omega_{R}$, respectively. This evolving state will include all the information currently stored by the given party, except for its secret key. Because we allow erasures, the current state does not include any information previously erased by this party.

We already stated that we only consider two kinds of attackers: sender impersonator $\mathcal{A}_{S}$ and receiver impersonator $\mathcal{A}_{R}$. The sender impersonator $\mathcal{A}_{S}$ will talk with an honest receiver $R$, while the receiver impersonator $\mathcal{A}_{R}$ will talk to an honest sender $S$. By assumption, there exists efficient simulators $\operatorname{Sim}_{R}$ and $\operatorname{Sim}_{S}$ for $\mathcal{A}_{S}$ and $\mathcal{A}_{R}$, respectively: the job of $\operatorname{Sim}_{R}$ is to simulate the behavior of $R$ when talking to $\mathcal{A}_{S}$, while the job of $\operatorname{Sim}_{S}$ is to simulate the behavior of $S$ when talking to $\mathcal{A}_{R}$. Moreover, the GUC security of $\Pi$ implies that $\operatorname{Sim}_{S}$ and $\operatorname{Sim}_{R}$ have to work given only oracle access to $R$ and $S$, respectively 4 In particular, this means that in each round $1 \leq i \leq r$,

- As long as neither $S$ nor $R$ is corrupted, $\operatorname{Sim}_{S}$ (resp., $\operatorname{Sim}_{R}$ ) will receive some arbitrary message $\alpha_{i}$ (resp., $\beta_{i}$ ) and must generate a "good-looking" response $\beta_{i}$ (resp., $\alpha_{i+1}$ ). Moreover, it must do so without knowledge of the secret keys $S K_{S}$ and $S K_{R}$, or any future messages $\alpha_{i+1}, \ldots$ (resp., $\left.\beta_{i+1}, \ldots\right)$.

- If $S$ (resp. $R$ ) is corrupted, $\operatorname{Sim}_{S}$ (resp., $\operatorname{Sim}_{R}$ ) will be given the secret $S K_{S}$ (resp., $S K_{R}$ ), and must then generate a "consistent-looking" internal state $\omega_{S}$ (resp., $\left.\omega_{R}\right)$ for the corresponding party at round $i$. The pair $\left(S K_{S}, \omega_{S}\right)$ (resp., $\left.\left(S K_{R}, \omega_{R}\right)\right)$ will then be given to the attacker and the environment.

From this description, we make our first key observation: as long as $S$ and $R$ are not corrupted, it is within the power of our attackers $\mathcal{A}_{S}$ and $\mathcal{A}_{R}$ to internally

${ }^{4}$ This is because, without loss of generality, $\mathcal{A}_{S}$ and $\mathcal{A}_{R}$ are simply the dummy parties forwarding the messages of the environment, and the simulator has to work for any environment. In fact, this property follows whenever there is an external "judge" with whom the adversary may interact when gathering evidence of protocol interactions. 
run the simulators $\operatorname{Sim}_{S}$ and $\operatorname{Sim}_{R}$, respectively. In particular, we can make meaningful experiments where $\mathcal{A}_{S}$ runs $\operatorname{Sim}_{S}$ against an honest receiver $R$, or $\mathcal{A}_{R}$ runs $\operatorname{Sim}_{R}$ against an honest sender $S$. Of course, a priori it is unclear what happens during these experiments, since $\operatorname{Sim}_{S}$ was only designed to work against attackers $\mathcal{A}_{R}$ who do not know $S K_{R}$ (as opposed to $R$ itself, who certainly knows it), and similarly for $\operatorname{Sim}_{R}$. The bulk of the proof consists of showing that such "unintended" usages of $\operatorname{Sim}_{S}$ and $\operatorname{Sim}_{R}$ nevertheless result in the "expected" behavior. We give a sequence of hybrid experiments which show that, without knowing the secret key of the sender, the simulator $\operatorname{Sim}_{S}$ can still successfully imitate the sender to an honest receiver, contradicting the soundness of identification. The details are given in the full version.

\section{Circumventing the Impossibility Result}

In this section, we discuss several positive results that circumvent the impossibility result of the previous section. We exhibit:

- A 1-message deniable authentication protocol tolerating adaptive corruptions, assuming a symmetric key infrastructure (i.e., a symmetric key shared between the sender and receiver);

- A 1-message deniable authentication protocol tolerating static corruptions in the PKI model;

- A 4-message protocol achieving a relaxed notion of deniable authentication, dubbed incriminating abort, and tolerating semi-adaptive corruptions in the PKI model. The protocol we give also satisfies the non-relaxed definition with respect to a static adversary.

Key exchange and deniable authentication can be reduced to each other, and so the results above also imply the feasibility of corresponding notions of deniable key exchange.

The first two results above are quite simple, and mainly serve to illustrate the gap between the simpler settings (symmetric keys and static corruptions) and the more realistic setting of public keys and adaptive corruptions. The third feasibility result is significantly more involved. We feel it represents an interesting and reasonable compromise between realistic modeling and feasibility.

Deniability with symmetric keys. Suppose for a moment that players have access to a symmetric key infrastructure; i.e., every pair of participants shares a uniformly random long-term key that is unknown to other participants. Then $S$ can authenticate a message $m$ to $R$ by appending a MAC (message authentication code) tag computed on the input (sid, $S, R, m$ ), where sid is a fresh random nonce. This is deniable roughly because the simulator for the protocol can make up a key for every pair of communicating players, and generate tags using the made-up key. In case of an adaptive corruption, the simulator can include the made-up key in the corrupted player's simulated memory contents.

This can be formalized in terms of the ideal functionalities $\mathcal{F}_{\text {auth }}$ and $\mathcal{F}_{k e}$. The SKI corresponds to granting every player of players one-time use of $\mathcal{F}_{k e}$, with key 
re-use modeled via the UC with joint state theorem [12. The use of a MAC shows that $\mathcal{F}_{\text {auth }}$ can be reduced to a one-time $\mathcal{F}_{k e}$. In fact, the converse is also true: one can realize $\mathcal{F}_{k e}$ by encrypting a key using a protocol that is secure against adaptive but passive adversaries (known as non-committing encryption [8]). The flows of this protocol can be authenticated using $\mathcal{F}_{\text {auth }}$ to make it resistant to active attacks.

Lemma $1\left(\mathcal{F}_{\text {auth }} \Longleftrightarrow \mathcal{F}_{k e}\right)$. If one way functions exist, then there exists a protocol that $U C$-realizes the natural multi-session extension of $\mathcal{F}_{\text {auth }}$ in the $\mathcal{F}_{k e}$ hybrid model, requiring only a single call to $\mathcal{F}_{k e}$. Conversely, if non-committing encryption exists, then there exists a protocol that $U C$-realizes $\mathcal{F}_{k e}$ in the $\mathcal{F}_{\text {auth }}$ hybrid model. These reduction hold even against adaptive adversaries.

Static security with a PKI. We now turn to the public-key model. For certain types of public keys, players can use a PKI to generate a symmetric key $k$ non-interactively. The idea of the protocol is then to use $k$ to compute tags on messages as above. The authenticity of the message is derived from the authentication inherent in the PKI. The non-interactive key generation is not adaptively secure, and so the resulting protocols are only secure against static adversaries.

For example, suppose we operate in a cyclic group $G$ generated by a generator $g$ where the Decisional Diffie-Hellman (DDH) assumption holds. If each party $P_{i}$ has a secret key $x_{i} \in \mathcal{Z}_{q}$ and a public key $y_{i}=g^{x_{i}}$, then $P_{i}$ and $P_{j}$ noninteractively share a key $k=g^{x_{i} x_{j}}=y_{i}^{x_{j}}=y_{j}^{x_{i}}$. Under the DDH asumption, $k$ looks like a random group element to an attacker who only knows the public keys $y_{i}$ and $y_{j}$. Either one of $P_{i}$ or $P_{j}$ can then use $k$ as a MAC key to authenticate messages to the other.

This type of key exchange is abstracted as non-interactive authenticated key exchange. We model the PKI via the registered keys with knowledge functionality $\mathcal{F}_{k r k}^{\Phi}$ (Figure [2) described in Section [2] (Key knowledge is necessary even for static security - see Proposition [2).

Theorem 3. Assuming the existence of non-interactive authenticated key exchange, there exists an efficient protocol $\Phi$ such that $\mathcal{F}_{\text {auth }}$ can be $U C$-realized in the $\mathcal{F}_{k r k}^{\Phi}$-hybrid model with respect to static adversaries.

In contrast, the impossibility result of Section 3 rules out adaptively secure GUC realizations. To the best of our knowledge, this is the first example of a task that cannot be realized with respect to adaptive adversaries, but can be achieved with respect to static adversaries.

\subsection{Deniability with Incriminating Abort}

Given the impossibility results of Section [3] and the possibility of PKI-based deniable authentication for static adversaries, it is natural to ask just how strong a notion of deniability can be achieved in the public key setting. We show here that one can guarantee deniability as long as (a) the protocol does not abort, and (b) there is one round during which neither the sender $S$ nor the receiver 
$R$ is adaptively corrupted. If the protocol aborts, the adversary can learn unsimulatable information depending on the secret keys of $S$ and $R$-potentially enough to prove that one of them was trying to talk to the other. We call this notion deniability with incriminating abort.

We refer to an adversary that makes no corruptions during some phase of the protocol run as semi-adaptive. In particular, such an adversary will not corrupt any players during the protocol's single vulnerable round. However, the restriction to semi-adaptive security is also necessary to make the notion of abort meaningful: a fully adaptive adversary could always ensure that a protocol does not abort by corrupting a party immediately before it complains. Semiadaptive security implies forward security; that is, a conversation that completes successfully is later deniable even if parties are forced to reveal the contents of their memories.

We phrase our results in terms of key exchange. This implies the corresponding feasibility results for authentication. However, forward security is especially meaningful for key exchange, because the protocol need only be run once, at setup time, for every pair of participants. If the key exchange protocol succeeds (with no adaptive corruption occurring during the protocol execution), then we can still use adaptively secure protocols realized in the $\mathcal{F}_{k e}$-hybrid model, and they will retain their adaptive security. In other words, the new protocol almost represents a deniable realization of $\mathcal{F}_{k e}$ : if we could somehow guarantee that the protocol never aborts, then it would GUC-realize $\mathcal{F}_{k e}$.

Modeling incriminating abort. We model the PKI via a shared, "registered keys with knowledge" functionality $\mathcal{F}_{k r k}^{\Phi}$ (Figure [2), as in the protocols for static adversaries. The key exchange with incriminating abort functionality $\mathcal{F}_{\text {keia }}$ is similar to $\mathcal{F}_{k e}$ except that the ideal-model adversary may explicitly request the protocol to abort, and in such a case the functionality will provide evidence that one of $S$ and $R$ was trying to talk to the other. It would be intuitively appealing to leak a single bit to the environment stating that a conversation occurred. We do not know of a way to ensure such limited leakage, and besides this gives up too much information: as we will see, our protocol only compromises deniability if the protocol aborts and one of $S$ or $R$ is corrupted at a later time. Instead, we parametrize $\mathcal{F}_{\text {keia }}$ with an "incrimination procedure" IncProc. In the case of an abort, $\mathcal{F}_{\text {keia }}^{\text {Inccoc }}$ allows the adversary to interact with $\operatorname{IncProc}\left(S K_{S}, \ldots\right)$, which essentially represents the potentially non-simulatable flows of the protocol. If the protocol doesn't abort, then a fresh symmetric key is distributed to $S$ and $R$ and nothing is leaked to the adversary. The functionality $\mathcal{F}_{\text {keia }}^{\text {IncProc }}$ is described in Figure [3]

The incrimination procedure may at first be hard to interpret, and so we highlight some properties of protocols that realize $\mathcal{F}_{\text {keia }}^{\text {IncProc }}$. First, if no abort occurs then the ideal protocol is forward secure, since the symmetric key is random and unconnected to other quantities in the protocol. Hence, if a protocol $\pi$ GUCrealizes $\mathcal{F}_{\text {keia }}^{\text {IncProc }}$ for any procedure IncProc then $\pi$ is forward-secure. Second, if an abort does occur and the adversary learns information, this information depends only on the sender's secret key and public information. Because secret 


\section{Functionality $\mathcal{F}_{\text {keia }}^{\text {Incroc }}$}

$\mathcal{F}_{\text {keia }}$, which is parameterized by an "incrimination procedure" IncProc, and a security parameter $\lambda$ proceeds as follows, when running in the $\mathcal{F}_{k r k}$-hybrid model with parties $S$ and $R$ (who have already registered secret keys $S K_{S}$ and $S K_{R}$, respectively) and adversary $\mathcal{S}$ :

1. Upon receiving a message of the form ( $S$, keyexchange, sid, $S, R, S K_{S}$ ) from party $S$, if there are no previous records, then record the value (keyexchange, sid, $S, R, S K_{S}$ ), mark $S$ "active", and a send public delayed output (keyexchange, sid, $S, R$ ) to $R$. (Otherwise, ignore the message.)

2. Upon receiving a message of the form ( $R$, keyexchange, sid, $S, R, S K_{R}$ ) from party $R$, if $R$ is not yet "active", mark $R$ as "active" and send a public delayed output (active, sid, $S, R$ ) to $S$. (Otherwise, ignore the message.)

3. Upon receiving a message of the form (setkey, sid, $S, R, k^{\prime}$ ) from $\mathcal{S}$, if $R$ is corrupt and $S$ is "active", then output (setkey, sid, $S, R, k^{\prime}$ ) to $S$ and $R$, and halt. If $R$ is "active" but not corrupt, then sample a fresh key $k \leftarrow\{0,1\}^{\lambda}$ and send the message (setkey, sid, $S, R, k$ ) to $R$. Furthermore, if $S$ is "active", then send the delayed message (setkey, sid, $S, R, k)$ to $S$ as well. In all cases, this completes the protocol, and the functionality halts.

4. Upon receiving a message of the form (abort, sid, $S, R$ ) from $\mathcal{S}$, if $S$ is "active", send (abort, sid, $S, R$ ) as a delayed message to $S$ and mark $S$ "aborted". If $R$ is "active", send (abort, sid, $S, R$ ) as a delayed message to $R$ (i.e., $\mathcal{S}$ need not notify either party that the protocol was aborted, and may still cause $R$ to output a key using a setkey message, but cannot cause $S$ to output a key once an abort has occurred).

5. Upon receiving a message of the form (incriminate, sid, $S$ ) from $\mathcal{S}$, if this is the first time receiving such a message and $S$ is currently "aborted" and honest, then run the procedure IncProc(sid, $\left.S, R, P K_{S}, P K_{R}, S K_{S}\right)$.

Fig. 3. The ideal functionality for Key Exchange with Incriminating Abort, parameterized by an incrimination procedure IncProc which runs only if the key exchange is aborted by the adversary

keys are useless in the ideal model, this has no impact on the security of other protocols. In particular, the incrimination information cannot be used to fake authenticated messages in other conversations, or to convince the environment that $S$ talked to anyone other than $R$. This last observation implies that not all incrimination oracles can be realized by real protocols: for example, if IncProc gives away the sender's secret key, then a real adversary would subsequently be able to fake arbitrary messages from the sender to other parties, contradicting the indistinguishability from the ideal model. We prove below that there exists a procedure IncProc for which $\mathcal{F}_{\text {keia }}^{\text {IncProc }}$ can, in fact, be realized.

Construction. At the core of our constructions for realizing $\mathcal{F}_{\text {keia }}$ is a chosenciphertext (CCA) secure variant of Dual Receiver Encryption (DRE) [15]. DRE allows anyone to encrypt a message to two parties with a single ciphertext, with the guarantee that attempts to decrypt a ciphertext by either of the two 


\begin{tabular}{|c|c|c|}
\hline \multirow[t]{2}{*}{$S$} & keyexchange $_{0}$, sid $, S, R$ & $R$ \\
\hline & $\psi_{1}=\operatorname{DREnc}\left(P K_{S}, P K_{R},\left\langle\right.\right.$ sid,$\left.\left.\eta_{R}\right\rangle\right)$ & $\eta_{R} \leftarrow\{0,1\}^{\lambda}$ \\
\hline $\begin{aligned}(\mathrm{pk}, \mathrm{sk}) & \leftarrow \mathrm{NCGen}() \\
\eta_{S} & \leftarrow\{0,1\}^{\lambda}\end{aligned}$ & $\psi_{2}=\operatorname{DREnc}\left(P K_{S}, P K_{R},\left\langle\right.\right.$ sid $\left.\left., \eta_{R}, \eta_{S}, \mathrm{pk}\right\rangle\right)$ & \\
\hline$k \leftarrow \operatorname{NCDec}(\mathrm{sk}, \gamma)$ & $\psi_{3}=\operatorname{DREnc}\left(P K_{S}, P K_{R},\left\langle\operatorname{sid}, \eta_{S}, \gamma\right\rangle\right)$ & $\begin{array}{c}k \leftarrow\{0,1\}^{\lambda} \\
\gamma \leftarrow \operatorname{NCEnc}(\mathrm{pk}, k)\end{array}$ \\
\hline
\end{tabular}

Fig. 4. A graphical illustration of Protocol $\Phi_{\text {dre }}$ for realizing $\mathcal{F}_{\text {keia }} . S$ and $R$ check consistency of each flow immediately upon receiving it; if the flow is not consistent, the protocol is aborted. Notation: (Gen, DREnc, DRDec) is a Dual Receiver Encryption scheme and (NCGen, NCEnc, NCDec, NCSim, NCEqv) is a Non-Committing Encryption scheme (see full version).

recipients will produce the same result. In our protocol, this means that certain actions can be simulated using either $S$ or $R$ 's secret key. We formally define CCA-secure DRE in the full version, and describe a DRE scheme that is similar to the plaintext-aware encryption of [21]. Our protocol also uses a 2-round noncommitting encryption (NCE) scheme [8].

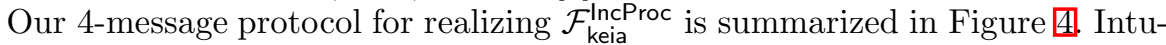
itively, the incrimination procedure IncProc will expect the adversary to supply a ciphertext that matches the form of the second flow $\left(\psi_{1}\right)$ in the protocol below, and will then use $S$ 's secret key to decrypt $\psi_{1}$ and compute a corresponding third flow $\left(\psi_{2}\right)$. The incrimination procedure hands $\psi_{2}$ to the adversary, along with the random coins used by a non-committing encryption scheme.

Notably, although we only realize $\mathcal{F}_{\text {keia }}^{\text {IncProc }}$ against a semi-adaptive adversary, the same protocol is also a statically secure realization of $\mathcal{F}_{k e}$. Therefore, we have achieved a strictly stronger notion of security than that achieved by the one-message protocol using NI-AKE and MACs, or HMQV. Honest parties are always guaranteed complete deniability when the protocol succeeds, and even if the protocol aborts, deniability is maintained until some future corruption of a party occurs. It is an open question whether this notion of deniability can be further improved upon.

Theorem 4. Assuming the existence of dual-receiver and non-committing encryption, the protocol $\Phi_{\text {dre }}$ in Figure 4

1. realizes $\mathcal{F}_{\text {keia }}^{\text {IncProc }}$ dre in the $\mathcal{F}_{k r k}^{\Phi_{d r e}}$-hybrid model with semi-adaptive security, for a suitable procedure IncProc $d r e$ (defined in the full version); and

2. realizes $\mathcal{F}_{k e}$ in the $\mathcal{F}_{k r k}^{\Phi_{d r e}}$-hybrid model with static security.

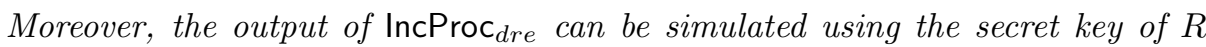
instead of $S$.

As mentioned above, realizing $\mathcal{F}_{\text {keia }}^{\text {IncProc }}$ is meaningful for any procedure IncProc. However, the particular incrimination procedure of IncProc has additional 
properties. First, it can be faked without knowing either secret key, and the fake distribution is indistinguishable from the real one to a distinguisher who knows neither key. Second, it can be simulated exactly using either of the secret keys. These properties together mean that $\Phi_{d r e}$ is statically secure (as stated in the theorem) and that even in the case of an abort with a subsequent corruption, it is only possible to incriminate one of the pair $\{S, R\}$, and not $S$ specifically.

Acknowledgments. We would like to thank Ran Canetti for many extremely illuminating discussions about composable authentication. We would also like to thank Yehuda Lindell, Rafael Pass and Amit Sahai for useful comments.

\section{References}

1. Barak, B., Canetti, R., Nielsen, J., Pass, R.: Universally composable protocols with relaxed set-up assumptions. In: FOCS, pp. 186-195. IEEE Computer Society, Los Alamitos (2004)

2. Bender, A., Katz, J., Morselli, R.: Ring signatures: Stronger definitions, and constructions without random oracles. In: Halevi, S., Rabin, T. (eds.) TCC 2006. LNCS, vol. 3876, pp. 60-79. Springer, Heidelberg (2006)

3. Borisov, N., Goldberg, I., Brewer, E.: Off-the-record communication, or, why not to use PGP. In: WPES, pp. 77-84. ACM, New York (2004)

4. Boyd, C., Mao, W., Paterson, K.G.: Deniable authenticated key establishment for internet protocols. In: Christianson, B., Crispo, B., Malcolm, J.A., Roe, M. (eds.) Security Protocols 2003. LNCS, vol. 3364, pp. 255-271. Springer, Heidelberg (2005)

5. Canetti, R.: Universally composable security: A new paradigm for cryptographic protocols. In: FOCS, pp. 136-145. IEEE Computer Society, Los Alamitos (2001)

6. Canetti, R.: Universally composable signatures, certification, and authentication. In: Computer Security Foundations Workshop, pp. 219-235. IEEE Computer Society Press, Los Alamitos (2004)

7. Canetti, R., Dodis, Y., Pass, R., Walfish, S.: Universally composable security with global setup. In: Vadhan, S.P. (ed.) TCC 2007. LNCS, vol. 4392, pp. 6185. Springer, Heidelberg (2007)

8. Canetti, R., Feige, U., Goldreich, O., Naor, M.: Adaptively secure multi-party computation. In: STOC, pp. 639-648. ACM, New York (1996)

9. Canetti, R., Fischlin, M.: Universally composable commitments. In: Kilian, J. (ed.) CRYPTO 2001. LNCS, vol. 2139, pp. 19-40. Springer, Heidelberg (2001)

10. Canetti, R., Kilian, J., Petrank, E., Rosen, A.: Black-box concurrent zeroknowledge requires (almost) logarithmically many rounds. SIAM J. Computing 32(1), 1-47 (2002)

11. Canetti, R., Kushilevitz, E., Lindell, Y.: On the limitations of universally composable two-party computation without set-up assumptions. J. Cryptology 19(2), 135-167 (2006)

12. Canetti, R., Rabin, T.: Universal composition with joint state. In: Boneh, D. (ed.) CRYPTO 2003. LNCS, vol. 2729, pp. 265-281. Springer, Heidelberg (2003)

13. Di Raimondo, M., Gennaro, R., Krawczyk, H.: Secure off-the-record messaging. In: WPES, pp. 81-89. ACM, New York (2005)

14. Di Raimondo, M., Gennaro, R., Krawczyk, H.: Deniable authentication and key exchange. In: Juels, A., Wright, R., De Capitani di Vimercati, S. (eds.) ACM Conf. Computer and Communications Security, pp. 400-409. ACM, New York (2006) 
15. Diament, T., Lee, H.K., Keromytis, A.D., Yung, M.: The dual receiver cryptosystem and its applications. In: Atluri, V., Pfitzmann, B., McDaniel, P.D. (eds.) ACM Conf. Computer and Communications Security, pp. 330-343. ACM, New York (2004)

16. Dolev, D., Dwork, C., Naor, M.: Nonmalleable cryptography. SIAM J. Computing 30(2), 391-437 (2000); Preliminary version in STOC 1991

17. Dwork, C., Naor, M.: Zaps and their applications. SIAM J. Computing 36(6), 1513-1543 (2007); Preliminary version in FOCS 2000

18. Dwork, C., Naor, M., Sahai, A.: Concurrent zero-knowledge. J. ACM 51(6), 851898 (2004); Preliminary version in STOC 1998

19. Dwork, C., Sahai, A.: Concurrent zero-knowledge: Reducing the need for timing constraints. In: Krawczyk, H. (ed.) CRYPTO 1998. LNCS, vol. 1462, pp. 442457. Springer, Heidelberg (1998); Full version available from the second author's webpage

20. Goldwasser, S., Micali, S., Rackoff, C.: The knowledge complexity of interactive proof systems. SIAM J. Computing 18(1), 186-208 (1989)

21. Herzog, J., Liskov, M., Micali, S.: Plaintext awareness via key registration. In: Boneh, D. (ed.) CRYPTO 2003. LNCS, vol. 2729, pp. 548-564. Springer, Heidelberg (2003)

22. Jakobsson, M., Sako, K., Impagliazzo, R.: Designated verifier proofs and their applications. In: Maurer, U.M. (ed.) EUROCRYPT 1996. LNCS, vol. 1070, pp. 143-154. Springer, Heidelberg (1996)

23. Jiang, S.: Deniable authentication on the internet. Cryptology ePrint Archive, Report 2007/082 (2007), http://eprint.iacr.org/

24. Katz, J.: Efficient and non-malleable proofs of plaintext knowledge and applications. In: Biham, E. (ed.) EUROCRYPT 2003. LNCS, vol. 2656, pp. 211-228. Springer, Heidelberg (2003)

25. Lim, M.-H., Lee, S., Park, Y., Moon, S.: Secure deniable authenticated key establishment for internet protocols. Cryptology ePrint Archive, Report 2007/163 (2007), http://eprint.iacr.org/

26. Nielsen, J.B.: Separating random oracle proofs from complexity theoretic proofs: The non-committing encryption case. In: Yung, M. (ed.) CRYPTO 2002. LNCS, vol. 2442, pp. 111-126. Springer, Heidelberg (2002)

27. Pass, R.: On deniability in the common reference string and random oracle model. In: Boneh, D. (ed.) CRYPTO 2003. LNCS, vol. 2729, pp. 316-337. Springer, Heidelberg (2003)

28. Di Raimondo, M., Gennaro, R.: New approaches for deniable authentication. In: Atluri, V., Meadows, C., Juels, A. (eds.) ACM Conf. Computer and Communications Security, pp. 112-121. ACM, New York (2005)

29. Richardson, R., Kilian, J.: On the concurrent composition of zero-knowledge proofs. In: Stern, J. (ed.) EUROCRYPT 1999. LNCS, vol. 1592, pp. 415-431. Springer, Heidelberg (1999)

30. Rivest, R., Shamir, A., Tauman, Y.: How to leak a secret. In: Boyd, C. (ed.) ASIACRYPT 2001. LNCS, vol. 2248, pp. 552-565. Springer, Heidelberg (2001)

31. Susilo, W., Mu, Y.: Non-interactive deniable ring authentication. In: Lim, J.-I., Lee, D.-H. (eds.) ICISC 2003. LNCS, vol. 2971, pp. 386-401. Springer, Heidelberg (2004)

32. Yao, A.C.-C., Yao, F., Zhao, Y., Zhu, B.: Deniable internet key-exchange. Cryptology ePrint Archive, Report 2007/191 (2007), http://eprint.iacr.org/ 